rev.relac.int.estrateg.segur.10(1):65-87,2015

\title{
EL CONCEPTO DE SEGURIDAD HUMANA EN LAS RELACIONES INTERNACIONALES•
}

\author{
Sandra Kanety Zavaleta Hernández**
}

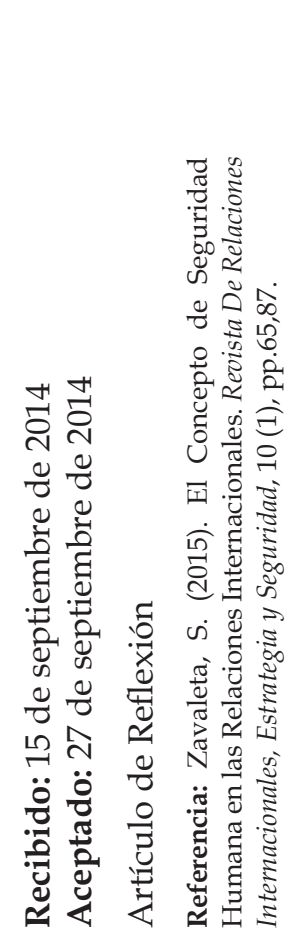

\section{RESUMEN}

Durante la década de los noventa, las transformaciones acontecidas en el orden internacional como resultado de la implosión de la Guerra Fría, así como la permanencia de estructuras y mecanismos de poder establecidos desde el orden de la segunda postguerra, darían pie a un complejo proceso de reestructuración de la dinámica mundial que habría de influir en la concepción de la seguridad. Con la instauración del Nuevo Orden Internacional, el paradigma unidimensional y unidireccional de seguridad, arraigado al conflicto y al elemento militar y predominante durante más de cuatro décadas, aprehendería en sus significaciones y entendimientos elementos multidimensionales y multidireccionales estrechamente vinculados a cuestiones inherentes al desarrollo. Desde un enfoque crítico de

Este artículo de reflexión surge de las investigaciones realizadas en el Instituto de Investigaciones Dr. José María Luis Mora y en la Universidad Nacional Autónoma de México.

** Posdoctora, Doctora en Ciencias Políticas y Sociales con orientación en Relaciones Internacionales, Maestra en Estudios en Relaciones Internacionales y Licenciada en Relaciones Internacionales por la Universidad Nacional Autónoma de México. Candidata a Investigadora del Sistema Nacional de Investigadores. kanetysandra@hotmail.com. 
la política internacional, el artículo tiene como objetivo así establecer las características coyunturales que en la dinámica de las relaciones internacionales coadyuvaron a la conformación de un concepto de seguridad antropocéntrico, de carácter multidimensional, universal, contextualizado e integrado por componentes interdependientes y mutuamente vulnerables: la seguridad humana.

Palabras clave: Seguridad Humana, Relaciones Internacionales, Seguridad Internacional, Seguridad Multidimensional, Post Bipolaridad.

\title{
THE CONCEPT OF HUMAN SECURITY IN INTERNATIONAL RELATIONS
}

\begin{abstract}
During the nineties, the transformations that took place in the international order as a result of the collapse of the Cold War, and the continuity of the power structures and mechanisms established since the second postwar period, would result in a complex process of restructuration of the global dynamics that had an impact in the design of security. With the beginning of the New World Order, the one-dimensional and one-directional security paradigm, rooted for more than four decades to conflict and military affairs, include in its meanings and understandings, multi-dimensional and multi-directional elements closely linked to issues related to development. From a critical approach to international politics, this paper aims to establish the relevant characteristics in international relations that contributed to develop an anthropocentric concept of security; multidimensional, universal, contextualized and integrated by interdependent and mutually vulnerable components: human security.
\end{abstract}

Keywords: Human Security, International Relations, International Security, Multidimensional Security, Post Bipolarity World.

\section{O CONCEITO DE SEGURANÇA HUMANA NAS RELAÇÕES INTERNACIONAIS}

\section{RESUMO}

Durante os anos noventa, as transformações ocorridas na ordem internacional, como resultado do colapso da Guerra Fria, e a continuidade das estruturas e dos mecanismos de poder estabelecidos desde o fim do segundo período pós-guerra, daria origem a um processo complexo de reestruturação das dinâmicas globais que influenciam a concepção de segurança. Com a introdução da Nova Ordem Mundial, o paradigma de segurança unidimensional e unidirecional, enraizado a os conflitos e ao elemento militar, predominante por mais de quatro 
décadas, aprehendería em seus significados e elementos entendimentos multidimensionais intimamente ligaos a questões relacionadas com o desenvolvimento. A partir de uma abordagem crítica da política internacional, este trabalho tem como objetivo estabelecer as características cíclicas que nas relações internacionais foram fundamentais para a formação de uma concepção de segurança antropocêntrica, com carateristicas multidimensionais, contextualizadas e integradas componentes caráter universais interdependentes e mutuamente vulneráveis: a segurança humana.

Palavras-chave: Segurança Humana, Relações Internacionais, Segurança Internacional, Segurança Multidimensional, Pós Bipolaridade.

\section{INTRODUCCIÓN}

La reconfiguración del orden internacional que trajo como resultado el colapso del mundo bipolar, daría pie a un lioso proceso de reestructuración de las relaciones internacionales (RRII) que habría de influir en la concepción de la seguridad, tanto en su perspectiva teórica como en la práctica.

La implosión de Unión de Repúblicas Socialistas Soviéticas -URSS- y del socialismo real, así como el fortalecimiento de la hegemonía de Estados Unidos -EE. UU. - y en consecuencia, del sistema capitalista, suscitarían un replanteamiento de extraordinaria complejidad en la definición de la seguridad. Con la disolución de la división Este-Oeste su concepción aprehendería otras dimensiones al liberarse de la estrechez analítica artificial a la que había sido reducida durante la confrontación bipolar, adquiriendo, por una parte, carácter multidimensional y, por otra, carácter multidireccional provenientes de un amplio espectro de actores o sujetos tradicionales, nuevos y atípicos y emergentes (Peña, 1998, p.77).

La reestructuración del sistema internacional permitió así la irrupción de una de las concepciones más integrales y complejas del término. En medio de un contexto de creciente discusión teórica y práctica alrededor de la seguridad, la convergencia de múltiples y complejos elementos coyunturales, característicos de la post bipolaridad, contribuyó a la construcción del concepto de seguridad humana.

\section{SURGIMIENTO DE UN CONCEPTO: EL CONTEXTO INTERNACIONAL}

El concepto de seguridad humana tuvo su gestación en el marco internacional de los años noventa y en el contexto político, social, económico e ideológico de la época. La disolución de la arquitectura bipolar y el fin del conflicto capitalismo-socialismo, junto con la caducidad de algunas de las estructuras de poder y de las estrategias militares edificadas bajo el esquema de 
Guerra Fría, permitirían la resignificación del paradigma tradicional hegemónico de seguridad, y con ello, el surgimiento del concepto de seguridad humana (Zavaleta, 2014).

La excesiva prioridad otorgada a la unidimensionalidad militar de la seguridad, materializada, por un lado, en la edificación de potentes alianzas militares a semejanza de cordones o cercos sanitarios de contención capitalista o socialista alrededor de todo el globo (como el Tratado Interamericano de Asistencia Recíproca, la Organización del Tratado del Atlántico Norte, el Tratado de Seguridad entre Australia, Nueva Zelandia y Estados Unidos, la Organización del Tratado del Sudeste de Asia, la Organización del Tratado de Asia Central y la Organización del Tratado de Varsovia) y, por otro lado, en los exorbitantes presupuestos destinados a la carrera armamentista entre las dos superpotencias, en aras de la preservación de la seguridad internacional, sería puesta en tela de juicio, en gran parte, por la creciente manifestación de fenómenos de carácter multidimensional -ya no sólo de carácter militar- que durante la confrontación Este-Oeste habían mantenido un perfil secundario (Zavaleta, 2014).

Paralelamente, el hecho de que regiones geopolíticamente importantes para el mantenimiento de la hegemonía de ambas potencias disminuyeran la función estratégica que les significó durante la Guerra Fría -lo que se traduciría de alguna u otra manera en una oportunidad, aparente al menos, para que los países que habían actuado de acuerdo a los intereses de las superpotencias, lo hicieran de acuerdo a los suyos- determinaría que la definición de amenazas y agendas de seguridad, asumieran dimensiones distintas a las establecidas e impuestas en el marco de la confrontación capitalismo vs socialismo.

En efecto, la implosión de Unión Soviética -US- y la preponderancia de EU.UU., traerían como consecuencia directa una (re)configuración internacional y con ello, el debilitamiento de conceptos y prácticas de seguridad arraigados a la visión tradicional.

Por un lado, la idea de la amenaza comunista, la percepción del holocausto nuclear y las concepciones de enemigo externo, contención, disuasión, destrucción mutua asegurada y equilibrio del terror - dominantes durante toda la Guerra Fría y manifestados a través de intervenciones militares en Afganistán, Angola, Checoslovaquia, Cuba, República Dominicana, Vietnam, etc. -, perdieron peso en la percepción generalizada de seguridad conduciendo a una gran efervescencia de cuestionamientos y críticas a las premisas unidimensionales y unidireccionales del arquetipo tradicional y contribuyeron al proceso de construcción de una concepción de seguridad vinculada con fenómenos de carácter económico, político, social, cultural, militar, etc., y con amenazas provenientes de varios actores, ya no sólo del Estado (Zavaleta, 2014).

Por otro, el cambio medular que sufrió la concepción de seguridad que el hegemón capitalista mantuvo durante la bipolaridad, mutando el eje alrededor del cual se había edificado el esquema tradicional que le dio preeminencia al componente militar, abrió la oportunidad para que otros temas -como narcotráfico, genocidios, regímenes no democráticos, derechos 
humanos, medio ambiente, entre otros-, fueran contemplados como prioridades en la agenda de seguridad estadounidense, facilitando la irrupción de concepciones integrales contentivas de diversos elementos ${ }^{1}$.

A la par de este proceso de desmantelamiento de los componentes centrales en la concepción tradicional de seguridad, durante los noventas se evidenciaron también procesos y coyunturas dentro de la dinámica mundial que favorecieron la aparición de un concepto más centrado en las personas y en sus comunidades que en el Estado y enfocado más al desarrollo que a las armas (PNUD, 1994).

La visibilización a nivel internacional de las devastaciones que tuvieron como efecto los conflictos en diferentes territorios, específicamente en los de menor desarrollo; el predominio de enfrentamientos de carácter intraestatal por encima de aquellos de carácter interestatal; el aumento del número de víctimas civiles frente a la cantidad de víctimas militares; la proliferación de actores no estatales interesados en temas diversos y, no menos importante, la manifestación cada vez mayor de las secuelas negativas de la implementación de las políticas neoliberales y de la expresión profunda de las crisis políticas, sociales y económicas en las regiones más pobres del mundo; coadyuvaron en conjunto al reconocimiento de amenazas o riesgos diversos, ya no sólo militares, en la agendas de seguridad.

La pobreza, el hambre, la marginación, la exclusión social, el deterioro ambiental, etc., serían consideradas, aunque con resistencia, amenazas contundentes a la seguridad de las personas; amenazas que hasta entonces no habían sido contempladas como prioritarias en los temas de seguridad de los Estados (Zavaleta, 2014).

Numerosos conflictos que habían permanecido congelados durante la Guerra Fría (Knigth, 2003) se patentaron con más fuerza que antes en el escenario mundial ${ }^{2}$. Para mediados de los noventas, habría casi un centenar de conflictos de índole diversa alrededor del mundo; la mayoría localizados en las regiones más pobres del planeta y todos relacionados directa o indirectamente con la injerencia de las potencias ${ }^{3}$ y con el pasado histórico de desarrollo desigual.

1. Es menester mencionar, sin embargo, que si bien el cambio de percepción en la definición de seguridad sería un fenómeno "aceptado globalmente", EE.UU. admitiría la inserción de diversas problemáticas y amenazas en su concepción como una estrategia de control más que de conciliación o consenso con el resto; una vez erradicado el "enemigo comunista", la potencia debía mantener su hegemonía a través de mecanismos encaminados al "combate" de "otras amenazas a su seguridad".

2. El origen de muchos de estos conflictos no reside necesariamente en el contexto bipolar. Muchos encuentran su génesis tiempo atrás, incluso antes de la dominación colonial. Sin embargo, y pese a que diversas pueden ser sus causas, todos captarían mayor atención internacional con la erradicación de la amenaza comunista y la mundialización de los medios de información.

3. Tanto de imperios europeos durante la colonización como de las dos superpotencias del mundo bipolar. 
Los conflictos vigentes en los ochentas y noventas en diferentes territorios, específicamente en los de menor desarrollo, como Angola, Bangladesh, Chad, Etiopía, Georgia, Irak, Líbano, Mozambique, Pakistán, Somalia, Sudán, Uganda, Zaire y, de manera particular, en Ruanda y Srebrenica en Bosnia, evidenciarían las devastaciones humanas, morales, económicas y sociales, que dejó como efecto la injerencia de las potencias en territorios considerados estratégicos para el fortalecimiento de los bloques capitalista y socialista durante la bipolaridad (Zavaleta, 2014).

Para finales de 1994, la masacre en Ruanda había cobrado la vida de más de un millón de tutsis de los cuales aproximadamente 300,000 serían niños. 500,000 personas sufrieron la mutilación de alguna parte de su cuerpo y miles de mujeres serían ultrajadas sexualmente. Se considera que el $75 \%$ de la etnia tutsi fue eliminada durante el genocidio (AWID, 2008); para mediados de 1995, el Ejército de la República Srpska bajo el mandato del General Ratko Mladic había asesinado a más de 8000 personas de la etnia bosnia. Aunque la limpieza étnica tuvo por objetivo asesinar a hombres musulmanes bosnios, entre las víctimas proliferaron también miles de mujeres y niños (OMS, 2002).

La magnitud destructiva, la difusión mediática y el impacto ocasionado en la opinión internacional de los genocidios ejecutados en Ruanda entre 1990 y 1994, y en Srebrenica en Bosnia y Herzegovina entre 1992 y 1995, conllevaron una gran efervescencia de cuestionamientos y críticas a las premisas realistas, estrechas, unidimensionales y unidireccionales del arquetipo de seguridad y contribuirían, en consecuencia, a la construcción de nociones con elementos conceptuales sistémicos, amplios, multidimensionales y multidireccionales

El fin del andamiaje bipolar permitió percibir también el predominio de enfrentamientos de carácter intraestatal por encima de los de carácter interestatal y el aumento del número de víctimas civiles en comparación con la cantidad de víctimas militares. De los 82 conflictos desarrollados entre 1989 y 1992, 79 se llevarían a cabo al interior de los países y únicamente tres se suscitarían entre Estados. La mayoría de estos enfrentamientos tendría lugar en países considerados de medio o bajo desarrollo. Durante 1993 hubo 52 conflictos de gran magnitud en 42 Estados y en otros 37 hubo conatos de violencia política. De esos 79 países, 65 corresponderían a países en desarrollo (PNUD, 1994). A principios del siglo XX, alrededor del 90\% de las víctimas de los conflictos eran militares; para finales del mismo periodo, el 90\% corresponderían a civiles. Más de la mitad de los enfrentamientos habían permanecido más de una década arrojando entre cuatro y seis millones de muertos de la población civil. Entre 1989 y 1992 perdieron la vida más de 1000 personas por año en ocho países: Afganistán, Angola, Filipinas, India, Perú, Somalia, Sudán y Sri Lanka (PNUD, 1994).

Por otro lado, la implementación del neoliberalismo, como teoría y práctica en las RRII, evidenció en los años noventa también la debilidad del Estado como garante de la seguridad de las poblaciones, y los costos sociales y económicos que ello trajo a la vida cotidiana de millones de personas en todo el mundo. 
En un contexto caracterizado por desajustes estructurales en el plano económico y severas críticas a la estatización de lo público y lo social, como esferas privilegiadas de acción del Estado de bienestar, se impulsó la construcción de propuestas basadas en el repliegue de las funciones interventoras y reguladoras que caracterizaban al Estado, para dar paso a las capacidades auto reguladoras del libre mercado. Todas estas transformaciones trastocaron referentes fundamentales necesarios para dotar al Estado de legitimidad y permitir el fortalecimiento de identidades colectivas que facilitaran, a su vez, reducir los espacios de incertidumbre, miedo e inseguridad sociales (Márquez, 1998).

El Estado Nación neoliberal adoptó así nuevas filosofías, nuevos discursos y naturalmente, nuevas prácticas que contradicen la propia esencia y razón de ser del Estado, mostrándose incapaz de dar respuesta a la complejidad de las demandas sociales y evidenciando con ello su imposibilidad de brindar a la población las condiciones de seguridad, salud, vivienda y educación necesarias para el goce de una vida estable y pacífica (Sánchez Noriega, 2011).

Lejos de proveer seguridad a sus poblaciones, los Estados elaboraron una amplia gama de discursos y de políticas provocando, manteniendo y llevando a la exaltación del individualismo, el quebranto de la sociabilidad (Sánchez Noriega, 2011) y, no menos importante, a la fragmentación de la legitimidad del aparato estatal. El Estado se volvió una amenaza tácita a la seguridad de quienes gobierna.

Estas características alrededor del Estado, permitieron, entre otras, la proliferación de un número significativo de actores no estatales, impulsados por motivaciones políticas, religiosas, económicas, sociales, culturales (Villanueva, 2002), así como su vertiginosa participación en la definición de temáticas de interés colectivo y de carácter holista, como desarrollo, medio ambiente, derechos humanos, salud pública, ayuda humanitaria, participación ciudadana, cuestiones de género, infancia, etc., fortaleciendo la idea de que la unidimensionalidad del concepto de seguridad debía replantearse para dar paso a interpretaciones más incluyentes.

Como consecuencia directa de la implementación de políticas de raigambre neoliberal y del quiebre del Estado, el fin de la bipolaridad evidenciaría como nunca antes, la fase más aguda de las crisis políticas, sociales y económicas en las regiones consideradas más pobres del mundo, visibilizando con ello las precarias condiciones en las que vivían millones de personas alrededor del planeta.

En efecto, la caducidad del orden bipolar permitió prestar más atención, por un lado, a los problemas inherentes a la división Norte-Sur (desarrollo-subdesarrollo) que a la confrontación Este-Oeste (capitalismo-socialismo) y, por otro, a las secuelas negativas que había dejado la implantación de modelos hegemónicos de desarrollo en gran parte del orbe, lo que condujo a que las problemáticas cotidianas derivadas del subdesarrollo fueran percibidas para muchos como amenazas importantes a su seguridad, facilitando la inserción de una plétora de temáticas variadas en los asuntos internacionales, mezcladas además entre sí. Las cuestiones de desarrollo serían percibidas entonces como cuestiones de seguridad. 
El orden mundial surgido en los noventas mostró así los estragos cotidianos en aquellos territorios que durante la Guerra Fría fueron del interés de las potencias en el mantenimiento del statu quo. La desigualdad y la polarización en diversos ámbitos del desarrollo, como la esperanza de vida, el alfabetismo o el ingreso, se harían más patentes que nunca.

Cuadro 1. Desigualdad en los noventas

\begin{tabular}{|c|c|c|c|}
\hline País & $\begin{array}{c}\text { Esperanza de vida al } \\
\text { nacer (años) } 1992\end{array}$ & $\begin{array}{l}\text { Tasa de alfabetismo } \\
\text { de adultos (\%) } 1992\end{array}$ & $\begin{array}{c}\text { PIB per capita (PPA }{ }^{4} \text { en } \\
\text { dólares) } 1991\end{array}$ \\
\hline Alemania & 75.6 & 99.0 & 19,770 \\
\hline Australia & 76.7 & 99.0 & 16,680 \\
\hline Canadá & 77.2 & 99.0 & 19,320 \\
\hline Estados Unidos & 75.6 & 99.0 & 22,130 \\
\hline Francia & 76.6 & 99.0 & 18,430 \\
\hline Japón & 78.6 & 99.0 & 19,390 \\
\hline Noruega & 79.6 & 99.0 & 17,170 \\
\hline Países Bajos & 77.2 & 99.0 & 16,820 \\
\hline Reino Unido & 75.8 & 99.0 & 16,340 \\
\hline Suecia & 77.7 & 99.0 & 17,490 \\
\hline Suiza & 77.8 & 99.0 & 21,780 \\
\hline Afganistán & 42.9 & 31.6 & 700 \\
\hline Burkina Faso & 47.9 & 19.9 & 666 \\
\hline Chad & 46.9 & 32.5 & 447 \\
\hline Gambia & 44.4 & 30.0 & 763 \\
\hline Guinea & 43.9 & 26.9 & 500 \\
\hline Guinea Bissau & 42.9 & 39.0 & 747 \\
\hline Mali & 45.4 & 35.9 & 480 \\
\hline Níger & 45.9 & 31.2 & 542 \\
\hline Sierra Leona & 42.4 & 23.7 & 1,020 \\
\hline Somalia & 46.4 & 27.0 & 759 \\
\hline
\end{tabular}

Fuente: elaborado por la autora con datos de PNUD.

Mientras que en EE. UU., Noruega, Japón, Suiza, Canadá, Francia o Alemania la esperanza de vida superaba en promedio los 75 años de edad y la tasa de alfabetización el 99\%; en

4. Paridad del Poder Adquisitivo (PPA) se refiere al tipo de cambio que refleja las diferencias de precios entre países y permite la comparación internacional de la cifra real de resultados e ingresos. En la tasa de PPA en dólares estadounidenses, 1 dólar estadounidense de PPA tiene el mismo poder adquisitivo en la economía nacional que 1 dólar estadounidense en Estados Unidos. 
Guinea Bissau, Sierra Leona, Afganistán, Gambia, Malí o Somalia, la población apenas lograría sobrevivir hasta los 44 años y lograr una alfabetización del 30\% (PNUD, 2009).

Entre 1990 y 1995, alrededor de 800 millones de personas en los países considerados de menor desarrollo carecieron de alimentos suficientes; 1,200 millones no tuvo acceso a agua potable; 800 millones no tuvieron acceso a servicios básicos de salud; la mortalidad materna era de 500 mujeres por cada 100,000 nacidos vivos; de los 500 millones que padecían malnutrición, 160 millones tenían menos de 5 años de edad; mientras que de los 840 millones de analfabetos, 110 millones eran niños (PNUD, 1994).

Cuadro 2. Estado de la pobreza. Millones de personas.1990-1995.

\begin{tabular}{|l|c|c|c|c|c|c|}
\hline \multicolumn{1}{|c|}{ Regiones } & $\begin{array}{c}\text { Adultos } \\
\text { analfabetos } \\
\mathbf{1 9 9 5}\end{array}$ & $\begin{array}{c}\text { Población } \\
\text { sin acceso } \\
\text { a servicios } \\
\text { de salud } \\
\mathbf{1 9 9 0 - 1 9 9 5}\end{array}$ & $\begin{array}{c}\text { Población } \\
\text { sin acceso } \\
\text { a agua } \\
\text { potable } \\
\mathbf{1 9 9 0 - 1 9 9 6}\end{array}$ & $\begin{array}{c}\text { Niños } \\
\text { menores de } \\
\mathbf{5} \text { años en } \\
\text { estado de } \\
\text { malnutrición } \\
\mathbf{1 9 9 0 - 1 9 9 6}\end{array}$ & $\begin{array}{c}\text { Tasa de } \\
\text { mortalidad } \\
\text { materna } \\
\text { (por } \\
\mathbf{1 0 0 0 0 0} \\
\text { nacidos) } \\
\mathbf{1 9 9 0}\end{array}$ & $\begin{array}{c}\text { Población } \\
\text { que se } \\
\text { estima no } \\
\text { sobrevivirá } \\
\text { hasta los 40 } \\
\text { años 90 }\end{array}$ \\
\hline $\begin{array}{l}\text { Países } \\
\text { árabes }\end{array}$ & 59 & 29 & 54 & 5 & 380 & 26 \\
\hline Asia Oriental & 167 & 144 & 398 & 17 & 95 & 81 \\
\hline $\begin{array}{l}\text { América } \\
\text { Latina y el } \\
\text { Caribe }\end{array}$ & 42 & 55 & 109 & 5 & 190 & 36 \\
\hline $\begin{array}{l}\text { Asia } \\
\text { Sudoriental y } \\
\text { el Pacífico }\end{array}$ & 38 & 69 & 162 & 20 & 447 & 52 \\
\hline $\begin{array}{l}\text { África } \\
\text { subsahariana }\end{array}$ & 122 & 205 & 249 & 28 & 971 & 124 \\
\hline $\begin{array}{l}\text { Todos los } \\
\text { países en } \\
\text { desarrollo }\end{array}$ & 842 & 766 & 1,213 & 158 & 471 & 507 \\
\hline
\end{tabular}

Fuente: PNUD, 1994.

En 1965, el PIB medio del 20\% más rico de la población mundial fue 30 veces mayor al del 20\% más pobre. Para 1990, esa diferencia se duplicó haciéndose de 60 veces (UNCTAD, 1997); para 1994 la relación entre el ingreso del 20\% más rico del mundo y el ingreso del 20\% más pobre era de 78 a 1, en comparación con 30 a 1 en 1960; para el mismo año, alrededor de 1,300 millones de personas registraron un ingreso de menos de un dólar diario; en Asia Meridional, Asia Oriental, Asia Sudoriental y el Pacífico se concentrarían 950 millones de pobres; en América Latina, 110 millones; y en África, 220 millones. 
Cuadro 3. Disparidades en el PIB (1960/1990) Índice Norte $=100$.

\begin{tabular}{|l|c|c|}
\hline \multicolumn{1}{|c|}{ Países de América Latina } & PIB per $\mathbf{1 9 6 0}$ & Real capita $\mathbf{1 9 9 0}$ \\
\hline Argentina & 53 & 34 \\
\hline Bolivia & 18 & 14 \\
\hline Chile & 49 & 46 \\
\hline Costa Rica & 34 & 33 \\
\hline El Salvador & 20 & 14 \\
\hline Guatemala & 26 & 21 \\
\hline Haití & 14 & 6 \\
\hline Honduras & 14 & 12 \\
\hline Jamaica & 29 & 24 \\
\hline México & 45 & 47 \\
\hline Nicaragua & 27 & 17 \\
\hline Perú & 33 & 20 \\
\hline Venezuela & 61 & 53 \\
\hline
\end{tabular}

\begin{tabular}{|c|c|c|}
\hline Países de África & PIB per 1960 & Real capita 1990 \\
\hline Argelia & 26 & 19 \\
\hline Burundi & 7 & 4 \\
\hline Chad & 12 & 3 \\
\hline Costa de Marfil & 16 & 10 \\
\hline Etiopía & 4 & 2 \\
\hline Ghana & 16 & 6 \\
\hline Kenya & 10 & 9 \\
\hline Madagascar & 16 & 5 \\
\hline Malí & 8 & 3 \\
\hline Mozambique & --- & ---- \\
\hline Nigeria & 18 & 9 \\
\hline República Centroafricana & 13 & 4 \\
\hline Ruanda & 8 & 4 \\
\hline Senegal & 18 & 11 \\
\hline Somalia & 14 & 5 \\
\hline Zambia & 18 & 7 \\
\hline
\end{tabular}

\begin{tabular}{|l|c|c|}
\hline \multicolumn{1}{|c|}{ Países de Asia } & PIB real1960 & Per capita $\mathbf{1 9 9 0}$ \\
\hline Bangladesh & 10 & 8 \\
\hline Filipinas & 18 & 16 \\
\hline India & 10 & 8 \\
\hline Irán & 31 & 31 \\
\hline Jordania & 21 & 19 \\
\hline Nepal & 9 & 7 \\
\hline Pakistán & 13 & 13 \\
\hline
\end{tabular}

Todas las cifras están expresadas en relación con el promedio del Norte, cuyo índice es 100. Cuanto menor es la cifra, mayor es la disparidad, y cuanto más se aproxima la cifra a 100, menor es la disparidad Fuente: Cuadro elaborado por la autora con datos de PNUD, 1994. 
En medio de todo este contexto de polaridades extraordinaria, de injusticia social, de agudización del subdesarrollo y de la inseguridad que ello traería consigo para millones de seres humanos alrededor del planeta se gestaría uno de los conceptos de seguridad más complejos, integrales y debatidos hasta ahora.

\section{LA SEGURIDAD HUMANA}

Si bien desde los setentas al interior de esferas académicas y de algunos organismos orientados particularmente a los estudios para la paz, el desarme y la resolución de conflictos, se generaron concepciones amplias de la seguridad y se definieron sus riesgos y amenazas en estrecha relación con fenómenos multidimensionales ${ }^{5}$ como así lo refieren, por ejemplo, las construcciones conceptuales de seguridad común ${ }^{6}$, seguridad societal ${ }^{7}$, seguridad global ${ }^{8}$,

5. Al respecto, una de las aportaciones académicas más reconocidas es la del politólogo noruego Johan Galtung quien ha vinculado las cuestiones de seguridad con la paz y la violencia, estudiadas, también, desde perspectivas multidimensionales. Formuladas dentro del contexto bipolar, las tesis de Galtung estarían orientadas al cuestionamiento de la formación de alianzas, particularmente militares, a la carrera armamentista y a los mecanismos de disuasión nuclear que como estrategias eran utilizadas por las dos superpotencias como elementos disuasorios de posibles enfrentamientos entre ambas. Bajo esta lógica, Galtung planteó una política alternativa de seguridad que en efecto conllevase a un estado de paz (o por lo menos de ausencia de guerra) y de seguridad global. La tesis de "cuatro caminos hacia la paz y la seguridad" plantearía que mediante 1) la resolución de los conflictos, 2) el equilibrio de poder, 3) el desarme y 4) políticas alternativas de seguridad, podrían superarse los diferentes grados de inseguridad (Galtung, 1984).

6. La seguridad común hace referencia a la posibilidad de que la gente viva en condiciones de paz y dignidad, tenga satisfechas sus necesidades alimentarias, cuente con un empleo digno y se desenvuelva en un ambiente sin pobreza e indigencia (Fernández, 2005). De esta forma, y de acuerdo con lo contenido en el Reporte de la Comisión Independiente sobre Asuntos de Desarme y Seguridad. Seguridad mundial. Un programa para el desarme, "en su búsqueda de seguridad, las naciones se deben esforzar por alcanzar objetivos más ambiciosos que la estabilidad basada en los armamentos... Otra forma aún más eficaz de garantizar la seguridad es aquella referente a la creación de procesos positivos que puedan conducir a la paz y al desarme. Es esencial que se cree un proceso irreversible, con un impulso de índole tal que todas las naciones cooperen para la supervivencia común" (Olof Palme, 1982).

7. El término seguridad societal tiene sus raíces en la Escuela de Copenhague y se refiere a la aceptación de las colectividades humanas como "objetos de referencia" de la seguridad. Teniendo como base componentes de carácter cultural, la seguridad societal hace alusión a la "habilidad de una sociedad para persistir en su carácter esencial bajo condiciones cambiantes y amenazas posibles o actuales. Más específicamente, se trata de la sostenibilidad, dentro de condiciones aceptables para la evolución, de los tradicionales patrones de lenguaje, cultura, asociación, identidad religiosa y nacional, y costumbre. La "seguridad societal" es, por tanto, un asunto de identidad" (Moller, 2000).

8. Desarrollado con amplitud en 1991 en el Informe denominado Responsabilidad Común en la Década de los Noventas. Iniciativa de Estocolmo sobre Seguridad Global y Gobernanza, el término seguridad global está estrechamente vinculado a la paz y al desarrollo, a la pobreza, al medio ambiente, la democracia, la demografía, los derechos humanos, la gobernanza mundial, las instituciones internacionales, entre otros. El valor del Informe, así como la concepción integral que de seguridad establece, radica en que su contenido fue resultado de los intereses colectivos 
o seguridad ampliada ${ }^{9}$, fue hasta la década de los noventas que se conformó el concepto de seguridad humana.

Así, una vez disuelta la pugna que caracterizó a las RRII por más de cuatro décadas, la visión tradicional, estrecha, unidimensional y unidireccional de seguridad arraigada al conflicto y al elemento militar, dominante en el orden bipolar (Peña, 1998) parecía perder peso mientras las perspectivas, multidimensionales y multidireccionales, ganaban apoyo.

En medio de un contexto de creciente debate teórico y práctico alrededor de la seguridad, caracterizado por la convergencia de importantes coyunturas formadas a partir del resultado de los cambios acontecidos en el sistema internacional, fue al interior de la Organización de las Naciones Unidas que la noción de seguridad adquirió su connotación humana.

En 1992, específicamente dentro del Informe del Secretario General de las Naciones Unidas, denominado Un programa para la paz, se hizo hincapié en la necesidad de eliminar la estrechez conceptual que caracterizó la definición de seguridad durante el enfrentamiento ideológico antagónico entre las dos superpotencias. Se pensó que "si la principal amenaza desaparecía, la agenda de seguridad podía incluir otros tipos de amenazas no contempladas hasta entonces" (Gomariz, 2004, 198).

A la letra, el Informe planteaba:

En los últimos meses se ha acrecentado la convicción, tanto en las naciones grandes como en las pequeñas, de que se ha vuelto a presentar una oportunidad de alcanzar la paz y la seguridad internacionales, hacer respetar la justicia y los derechos humanos... Esa oportunidad no debe desperdiciarse. La Organización no debe volver a caer jamás en la impotencia a que se vio sometida en el período que ahora ha quedado atrás.

de cuatro Comisiones importantes en la materia: la Comisión Norte-Sur (encabezada por Willy Brandt), la Comisión Independiente de Desarme y Seguridad (encabezada por Olaf Palme y encargada de definir seguridad común, citada arriba), la Comisión Brundtland y la Comisión Sur. (Para mayor referencia sobre el término, se sugiere consultar también Dahlgren, H., 1992).

9. Atribuida también a la Escuela de Copenhague, el concepto de la seguridad ampliada distingue cinco dimensiones ampliadas de seguridad -militar, política, económica, societal y ambiental-y varios objetos de referencia (seguridad ante quién) y los niveles de interacción o análisis (profundización: internacional, regional, nacional, grupos domésticos organizados o movimientos sociales, familias e individuos). Mientras que desde la perspectiva de seguridad tradicional el dilema de seguridad entre Estados en conflicto representa una amenaza real, las amenazas sociales, energéticas, alimentarias, de salud y de bienestar pueden generar, desde la perspectiva de seguridad ampliada, un "dilema de supervivencia" en regiones de alta vulnerabilidad (Oswald, 2009). 
Las fuentes de los conflictos y las guerras son hondas y multifacéticas... En los últimos años se ha derrumbado una inmensa barrera ideológica que durante decenios fue fuente de desconfianza y hostilidad; han caído también los terribles instrumentos de destrucción que eran sus compañeros inseparables. A pesar de que las cuestiones que separan a los Estados del Norte y del Sur se hacen cada vez más agudas... el mejoramiento de las relaciones entre los Estados del Este y el Oeste brinda nuevas posibilidades para conjurar con éxito las amenazas que se ciernen sobre la seguridad común.

El concepto de la paz no es difícil de comprender; el de la seguridad internacional, en cambio, es más complejo, porque también en su caso se plantea todo un mosaico de contradicciones. A pesar de que las potencias nucleares han comenzado a negociar acuerdos de reducción de armamentos, la proliferación de armas de destrucción en masa amenaza con aumentar y en muchas partes del mundo continúan acumulándose los armamentos convencionales. Al tiempo que se reconoce que el racismo es una fuerza destructiva y se desarticula el apartheid, surgen nuevas tensiones raciales que buscan expresión en la violencia. El avance tecnológico transforma la naturaleza y las esperanzas de vida en todo el mundo. La revolución de las comunicaciones ha unido al mundo en cuanto a conciencia, aspiraciones y una mayor solidaridad contra la injusticia. Sin embargo, el progreso también entraña nuevos riesgos para la estabilidad: daños ecológicos, la destrucción de la vida de la familia y la comunidad, y una mayor intrusión en la vida y los derechos de los individuos.

No puede permitirse que esta nueva dimensión de la inseguridad eclipse los devastadores y constantes problemas del desenfrenado crecimiento de la población, de la carga aplastante de la deuda, de las barreras que se oponen al comercio, de las drogas y de las diferencias cada vez mayores entre ricos y pobres. Cunden la pobreza, las enfermedades, el hambre, la opresión y la desesperación... Se trata de elementos que, a la vez, son fuente y consecuencia de conflictos que exigen una atención incesante y un alto grado de prioridad en las actividades de las Naciones Unidas. Los agujeros de la capa de ozono pueden entrañar para la población afectada peligros mayores que los de un ejército hostil. La sequía y las enfermedades pueden diezmar a la población con la misma crueldad que las armas de la guerra. Es por ello que en estos momentos de renovada oportunidad, los esfuerzos de la Organización por consolidar la paz, la estabilidad y la seguridad deben englobar temas que trascienden las amenazas militares, para que sea posible romper las cadenas de los conflictos y las guerras que han caracterizado al pasado (Boutros-Ghali, 1992).

En sintonía, un año más tarde, el Informe sobre Desarrollo Humano del Programa de Naciones Unidas para el Desarrollo estableció que el concepto de seguridad debía evolucionar de tal manera que de basarse exclusivamente en la seguridad nacional se apoyara mucho más en la seguridad de la gente, pasando entonces de la seguridad a través del armamentismo a la seguridad mediante el desarrollo humano y de la seguridad territorial a la seguridad alimentaria, en el empleo y el medio ambiente (UNDP, 1993). 
Hoy día es necesario revisar radicalmente muchos conceptos arcaicos. Debe reinterpretarse la seguridad como una seguridad para la gente y no una seguridad para el territorio. El desarrollo debe centrarse en la gente y no la gente en el desarrollo... El concepto de seguridad debe cambiar, evolucionando de tal manera que de basarse exclusivamente en la seguridad nacional pase a destacar mucho más la seguridad de la gente, de la seguridad mediante el armamentismo hacia la seguridad mediante el desarrollo humano, de la seguridad territorial a la seguridad alimentaria, en el empleo y en el medio ambiente.

En 1994, en el Informe Un programa para la Cumbre Mundial de Desarrollo Social, específicamente en el apartado segundo "Nuevas dimensiones de la seguridad humana", el PNUD profundizaría en el carácter multidimensional de la seguridad mediante de la inclusión de temáticas diversas que abordarían cuestiones políticas, sociales, culturales, económicas, ambientales, entre otras, denotando, aún más, un alejamiento de la visión estatocéntrica, militar y territorial del paradigma tradicional de seguridad. Se consideraría entonces que la paz, la protección al medio ambiente, la vigencia de los derechos humanos, la integración social, podrían ser constituidas sólo dentro de un marco de desarrollo sostenible conducente a la seguridad de los seres humanos (PNUD, 1994). De esta manera, el documento expresó:

...el concepto de seguridad ha sido interpretado en forma estrecha durante demasiado tiempo: en cuanto a seguridad del territorio, contra la agresión externa, o como seguridad mundial frente a la amenaza de un holocausto nuclear. Las superpotencias estaban trabadas en una lucha ideológica, librando una Guerra Fría en todo el mundo. Los países en desarrollo, que habían logrado su independencia sólo recientemente, tenían sensibilidad respecto de cualquier amenaza, real o percibida, a su frágil identidad nacional. Se dejaban de lado las preocupaciones legítimas de la gente común que procuraba tener seguridad en su vida cotidiana. Para muchos, la seguridad simbolizaba la protección contra la amenaza de la enfermedad, el hambre, el desempleo, el delito, el conflicto social, la represión política y los riesgos del medio ambiente.... Para la mayoría de las personas, el sentimiento de inseguridad se debe más a las preocupaciones acerca de la vida cotidiana que al temor de un cataclismo en el mundo... son éstas las preocupaciones que están surgiendo acerca de la seguridad humana.

Como puede verse, la seguridad humana es una concepción amplia, integral, multidimensional y multidireccional de la seguridad. Se centra en las personas más que en el Estado y se constituye a partir de las necesidades humanas y de las capacidades que las personas posean para procurarse la mejor vida posible. En este sentido, la seguridad, desde su adjetivación humana, no es sólo un asunto reducido a las relaciones entre Estados o a cuestiones de interés estatal; implica también una connotación individual y comunitaria. No sólo está relacionada con el orden público y el cumplimiento de las leyes, sino que abarca otras dimensiones de la existencia del ser humano y de la relación que éste mantiene con su entorno natural y social. No hace referencia únicamente a la protección sino que además se apoya en la prevención y en la habilitación de las personas para valerse por sí mismas (Fernández, 2005). 
En definitiva, la seguridad humana no es una preocupación por las armas, por la defensa del Estado o por la protección de las fronteras políticas; es mejor dicho una preocupación por la vida y dignidad de las personas (Zavaleta, 2014).

Al intentar romper con el arquetipo unidimensional y unidireccional de seguridad predominante en el mundo bipolar, la seguridad humana pretende ser una categoría de carácter holista alejada de una noción defensiva y limitante al territorio y al elemento militar (Villanueva, 2002).

En este sentido, desde su origen la seguridad humana sería concebida con carácter multidimensional o multisectorial; integrada a partir de componentes interdependientes e indivisibles apoyados, a su vez, en la noción de vulnerabilidad mutua; con alcance universal pero al mismo tiempo contextualizado; y construida bajo un enfoque antropocéntrico.

\section{CARACTERÍSTICAS DE LA SEGURIDAD HUMANA}

Concepción antropocéntrica: La seguridad humana se centra en las personas. Desde su nacimiento, los objetivos, elementos e instrumentos que la componen están orientados a la forma en la que la gente vive y respira en la sociedad, la libertad con que puede ejercer diversas opciones, el grado de acceso a las oportunidades sociales y a la vida en conflicto o en paz (PNUD, 1994). La noción de seguridad humana se relaciona con el clamor de las personas, cualquiera que sea el lugar que habita, para tener calidad de vida adecuada, satisfacer sus aspiraciones y dar respuestas a sus incertidumbres inmediatas (Alvear, 2001).

Como concepción integral, la seguridad humana intenta responder a interrogantes de tipo cotidiano como si las personas tienen acceso a suficiente y adecuado alimento, si cuentan con algún ingreso, si son víctimas de represión, si se sienten seguras en las calles, si se desenvuelven en un medio natural sano, si cuentan con servicios educativos o de salud apropiados o si pueden procurar su capital emocional ${ }^{10}$.

10. El capital emocional difiere de otras formas de capital porque en la medida en que protege y brinda satisfacción a las personas, obtenerlo es un fin es sí mismo, más allá de la productividad alguna que pudiera producir. La supresión de una vida desagradable e incluso miserable por la capacidad de realizarse, ser feliz, vivir en justicia y equidad debe ser la prioridad en el capital emocional. Ello se entiende bajo la lógica de que al ser la vida humana el centro de atención de la seguridad, la salud y la armonía emocional y mental son cuestiones humanas prioritarias puesto que las emociones intervienen naturalmente en las acciones colectivas sociales. Si bien somos seres racionales también somos seres emocionales. La tristeza, la ira, el odio, el miedo, la depresión social, etc. son emociones que llevadas a un extremo patológico pueden ser amenazas críticas a la seguridad humana. Protegernos como individuos se vuelve sumamente importante porque ello escapa a la perspectiva de seguridad pública y de seguridad nacional (Baena, G. y Balbi, E., 2006). 
Colocar como eje a los individuos, hacer de las personas -y no de los Estados- el objeto principal de la seguridad, implica la consideración de un amplio espectro de condiciones o amenazas a la supervivencia, que podrían no ser prioritarias dentro de los esquemas de la seguridad. Si bien el Estado sigue siendo su proveedor fundamental, debe reconocerse que a menudo no efectúa sus obligaciones en materia de seguridad e incluso puede llegar a ser una fuente de amenazas para su propia población. Es por ello que el paradigma de seguridad humana considera que la atención debe ser desplazada de la seguridad del Estado a la seguridad de las personas (CHS, 2003). En efecto, la seguridad humana se constituye de las capacidades de la gente para hacerse de una vida más plena basándose en la participación activa de los individuos para lograr dichas opciones; la consecución de la seguridad humana no se refiere únicamente a la protección de la persona, sino a la facilitación de los medios para valerse por sí misma (CHS, 2003). En este sentido, la seguridad del Estado y la seguridad de la gente no deberían concebirse como opuestas sino como complementarias.

Concepción universal: La seguridad humana asevera que la seguridad es derecho, obligación y competencia de todos. A diferencia del paradigma tradicional, la seguridad humana considera que la seguridad puede ser una condición humana general, lo que reafirma que no necesariamente debe existir inseguridad en unos cuantos para que otros se sientan seguros. La seguridad, por tanto, es compartida y universal por ser propósito, preocupación e interés de todos los individuos. "Es pertinente a la gente de todo el mundo, tanto en países ricos como en países pobres" (PNUD, 1994, 25). Es competencia de todos porque en la totalidad del planeta existen riesgos o peligros que, si bien no son percibidos de igual manera en un lugar como en otro, en todos pueden vulnerar la seguridad de las personas en la cotidianidad de sus vidas.

Al mismo tiempo, puede percibirse que si la seguridad de las personas es amenazada en cualquier parte del mundo es muy probable entonces que todos los espacios resulten directa o indirectamente involucrados (PNUD, 1994). Al ser amenazas compartidas universalmente, la pobreza, la desintegración social, la contaminación ambiental, las migraciones, la violencia, etc., no pueden ser concebidos como fenómenos aislados ni pueden circunscribirse a los espacios geográficos.

Es decir, la seguridad y la inseguridad se encuentran vinculadas en todos los niveles del sistema; esto es, la seguridad en un nivel micro puede verse amenazada por la inseguridad padecida en el nivel macro y la seguridad del conjunto podría ser afectada por la inseguridad en los niveles micros (Nef, 2001). La seguridad humana entiende así que las amenazas existentes dentro de un espacio determinado pueden llegar a extenderse y tener repercusiones en la seguridad de otras latitudes. En este orden de ideas, las vulnerabilidades no podrían ser tratadas aislada, individual o fragmentadamente; por el contrario, el control, la erradicación e incluso la prevención de ellas deben estar basadas en mecanismos integrales, conjuntos, cooperativos, multiactorales y multisectoriales. 
Concepción multidimensional, multisectorial: Una de las más notables aportaciones de la noción de seguridad humana ha sido la atribución de carácter multidimensional que otorga a la significación de amenazas. En el afán de romper con la estrechez del paradigma unidimensional de la seguridad tradicional, esta concepción comprende que a las inseguridades convencionales, vinculadas intrínsecamente al elemento estatocéntrico y al factor militar, pueden sumarse otras tantas, de igual o mayor importancia, derivadas de elementos de orden político, social, cultural, económico, ambiental, entre otros. Al mismo tiempo, la seguridad humana comprende que la diversidad de amenazas sólo puede enfrentarse por medio de mecanismos o instrumentos igualmente diversos a través de redes o vínculos entre los distintos actores y en múltiples sectores.

Concepción interdependiente, indivisibles y de vulnerabilidad mutua: La seguridad humana está constituida por elementos interdependientes e indivisibles. La degradación ambiental, el hambre endémica, la diseminación de enfermedades, los actos terroristas, etc., trascienden fronteras dejando de ser competencia de una sola comunidad, de un sólo país o una sola región. Al componerse de diferentes esferas, la seguridad humana adquiere carácter indivisible porque las amenazas que afecten a una de estas dimensiones indudablemente tendrán efectos o repercusiones en las demás. Asimismo, se vuelve interdependiente porque el progreso de una esfera aumenta las posibilidades de lograr progreso en otra y el fracaso en una aumenta también el riesgo de que la otra fracase. La alteración o impacto que sufra alguno de los componentes afectará a todos las demás; de igual manera, el menoscabo de seguridad humana en una latitud se propagará, irremediablemente, a otros espacios.

Todas y cada una de las amenazas a la seguridad de los individuos son resultado de disfunciones internas de regímenes ambientales, políticos, culturales, económicos, etc., que afectan tanto los espacios domésticos como los globales. Debido al carácter interdependiente e indivisible entre estos espacios, las disfunciones que puedan existir en un subsistema tenderán a reproducirse en otros subsistemas conexos. En este sentido, la seguridad humana se asienta en la noción de vulnerabilidad mutua al considerar que dentro de un sistema global interconectado, la fortaleza o solidez del conjunto -incluyendo sus componentes más desarrollados y aparentemente mejor protegidos- se encuentran condicionados por sus eslabones más débiles. Así, la vulnerabilidad mutua se constituye de disfunciones yuxtapuestas vinculadas intrínsecamente a circuitos de causalidad múltiple; es decir que, mientras exista vulnerabilidad o inseguridad en algunos sectores del conjunto, todo y todos, en mayor o menor grado, se encuentran vulnerables (Nef, 2001). Los ámbitos individual, familiar, comunitario, estatal, regional y global se influyen dialécticamente.

Concepción contextualizada: Aunque en efecto la seguridad humana hace énfasis en el carácter universal de sus componentes -sosteniendo la existencia de amenazas compartidas de consecuencias generales- también comprende que las inseguridades pueden variar considerablemente de un individuo a otro, de una comunidad a otra, de un Estado a otro. 
Siendo un enfoque flexible, amplio y dinámico, por medio de la identificación de necesidades o inseguridades específicas dentro de un espacio determinado, la seguridad humana intenta aportar soluciones contextualizadas que respondan adecuadamente a situaciones o realidades particulares (UNTFHS, 2009). "Las amenazas en contra de la seguridad humana así como los recursos disponibles para afrontarlas varían significativamente a lo largo y al interior de las diferentes regiones y países del planeta... La sociedad y sus miembros reconocen, dan nombre, explicaciones y prioridades a las amenazas. Los reconocimientos y explicaciones, es decir la codificación de éstas, son socialmente relativos. En cualquier caso, no hay mecanismo de seguridad posible para amenazas no reconocidas ni codificadas socialmente... Las certezas, peligros y riesgos, así como los correspondientes mecanismos de seguridad están distribuidos desigualmente. La sociedad privilegia ciertas amenazas, ciertos peligros y riesgos a la hora de asignar las seguridades" (Kornblitch, 2001, 342).

\section{COMPONENTES DE LA SEGURIDAD HUMANA}

Tradicionalmente, la significación de la seguridad y la definición de sus amenazas han estado sustentadas en un carácter defensivo, de dimensiones estatocéntricas y definidas bajo elementos de orden militar. Aunque en efecto los conflictos entre Estados, los conflictos armados, las intervenciones militares, la violación de fronteras, entre otros, son vigentes y pueden llegar a dificultar e incluso imposibilitar la seguridad de muchas personas, también es cierto que para otras tantas la sensación de seguridad puede encontrarse en un empleo bien remunerado, en servicios adecuados de salud, o en tener alimento en cantidad y calidad suficientes; y verse amenazada, por ejemplo, por el hambre, la pobreza, la enfermedad, algún desastre medioambiental, etc.

Para la seguridad humana, entonces, los factores de (in)seguridad actuales son consecuencia de la concomitancia de multiplicidad de actores y elementos relacionados con variables sociales, culturales, políticas, económicas, militares, etc. Al tener como pilares a la libertad frente al miedo (freedom from fear) y a libertad frente a la necesidad (freedom for need), la seguridad humana hace referencia entonces a la percepción de temor respecto de la violencia -en donde se encuentran contemplados aspectos relacionados con ésta, como los conflictos armados, las intervenciones militares, el crimen organizado, las guerras, entre otros-, pero también respecto de elementos que están relacionados con el desarrollo -en donde se involucran aspectos inherentes a la alimentación, la salud, el empleo, la inclusión social, etc.-.

Bajo esta lógica, la seguridad humana podría verse vulnerada desde múltiples aristas y es en este sentido que intenta agrupar o clasificar sus posibles amenazas en distintas pero indivisibles y enlazadas categorías.

El acceso y calidad de los alimentos, la desnutrición, malnutrición o la subnutrición, el acceso a servicios de salud en cantidad y calidad suficientes, personal médico capacitado, acceso 
a fuentes de agua potable, esperanza de vida, pobreza humana, ingreso económico, desempleo, conflictos étnicos, exclusión social, tortura, terrorismo de Estado, persecución política, migración medioambiental, contaminación atmosférica, cambio climático, entre muchos otros, pueden ser así considerados factores e indicadores de seguridad o inseguridad humana.

Cuadro 4. Componentes e indicadores de la seguridad humana.

\begin{tabular}{|c|c|}
\hline Componente & Indicador \\
\hline $\begin{array}{c}\text { Seguridad } \\
\text { alimentaria }\end{array}$ & $\begin{array}{l}\text { Acceso a alimentos } \\
\text { Calidad de los alimentos } \\
\text { Índice de desnutrición y malnutrición } \\
\text { Índice de producción de alimentos per capita } \\
\text { Nivel de dependencia respecto a la importación y exportación de alimentos } \\
\text { Oferta diaria de calorías } \\
\text { Proporción entre alimentos y población } \\
\text { Otros }\end{array}$ \\
\hline $\begin{array}{l}\text { Seguridad } \\
\text { en la salud }\end{array}$ & $\begin{array}{l}\text { Acceso a servicios de salud } \\
\text { Gasto per capita en salud } \\
\text { Gasto público y privado en salud } \\
\text { Índice de esperanza de vida } \\
\text { Número de personal médico capacitado en función con el número de } \\
\text { habitantes. } \\
\text { Población con acceso a fuentes de agua potable } \\
\text { Porcentaje de la población inmunizada } \\
\text { Porcentaje de niños menores de cinco años con peso inferior al normal } \\
\text { Otros }\end{array}$ \\
\hline $\begin{array}{l}\text { Seguridad } \\
\text { económica }\end{array}$ & $\begin{array}{l}\text { Exportaciones e importaciones } \\
\text { Îndice de Pobreza Humana } \\
\text { Número de empleos respecto a la población económicamente activa } \\
\text { Paridad del Poder Adquisitivo (PPA) } \\
\text { Paridad o disparidad en los niveles de ingreso } \\
\text { PIB per capita } \\
\text { Tabulador del salario mínimo } \\
\text { Tasa de desempleo } \\
\text { Otros }\end{array}$ \\
\hline $\begin{array}{l}\text { Seguridad } \\
\text { personal }\end{array}$ & $\begin{array}{l}\text { Amenazas del Estado (tortura física, persecución política) } \\
\text { Amenazas provenientes de otros Estados (guerra) } \\
\text { Amenazas provenientes de otros grupos de la población (tensión étnica, tensión } \\
\text { religiosa, tensión racial) } \\
\text { Amenazas de individuos o pandillas contra otros (delincuencia, violencia } \\
\text { callejera) } \\
\text { Amenazas contra las mujeres (violaciones, violencia doméstica) } \\
\text { Amenazas contra los niños (maltrato físico o psicológico) } \\
\text { Amenazas contra la propia persona (suicidios, uso de estupefacientes) } \\
\text { Otros }\end{array}$ \\
\hline
\end{tabular}




\begin{tabular}{|c|c|}
\hline Componente & Indicador \\
\hline $\begin{array}{l}\text { Seguridad } \\
\text { comunitaria }\end{array}$ & $\begin{array}{l}\text { Conflictos étnicos } \\
\text { Conflictos sociales } \\
\text { Desplazados internos } \\
\text { Discriminación } \\
\text { Exclusión / inclusión social } \\
\text { Mantenimiento / pérdida de tradiciones } \\
\text { Marginación } \\
\text { Refugiados } \\
\text { Otros }\end{array}$ \\
\hline $\begin{array}{l}\text { Seguridad } \\
\text { política }\end{array}$ & $\begin{array}{l}\text { Gasto militar en función del gasto a salud, educación, otros. } \\
\text { Número de fuerzas armadas } \\
\text { Número de refugiados y desplazados internos } \\
\text { Represión, persecución, extorsión, censura, tortura } \\
\text { Respeto o violación a los derechos humanos. } \\
\text { Otros }\end{array}$ \\
\hline $\begin{array}{l}\text { Seguridad } \\
\text { ambiental }\end{array}$ & $\begin{array}{l}\text { Aprovechamiento adecuado o inadecuado de los recursos naturales. } \\
\text { Cambio climático } \\
\text { Consumo de combustible o electricidad per capita } \\
\text { Deforestación } \\
\text { Desertificación } \\
\text { Disponibilidad de agua } \\
\text { Emisión de contaminantes } \\
\text { Número de zonas ecológicas } \\
\text { Salinización } \\
\text { Otros }\end{array}$ \\
\hline
\end{tabular}

Fuente: elaborado por la autora con datos de PNUD, 1994 y de otros estudios sobre seguridad humana desarrollados por ella.

\section{CONCLUSIÓN}

La prioridad históricamente otorgada al carácter restringido en la concepción de seguridad en las RRII ha obedecido al sistema de dominación imperante de la dinámica mundial, generado prácticamente desde la propia configuración del orden bipolar.

Al término de la Segunda Guerra Mundial, la amenaza de guerra, el holocausto nuclear, la conformación de coaliciones de carácter militar, las intervenciones bélicas en múltiples territorios, así como la carrera armamentista figurarían como los principales elementos constitutivos en la significación de seguridad.

Una vez terminada la bipolaridad pudo ocurrir la emergencia de un concepto que, contrariamente al paradigma predominante, estaría constituido por elementos de carácter multidimensional, multidireccional, integrales y complejos. 
Una mayor visibilidad de los conflictos y la mutación en sus características, la emergencia de actores atípicos con diversidad de demandas, la evidencia de las secuelas de los modelos de desarrollo hegemónicos en los menos desarrollados, entre otros factores coadyuvaron a situar en las agendas de seguridad una plétora de prioridades y temas que durante el enfrentamiento bipolar mantuvieron un papel menor.

Dadas las coyunturas que propiciaron el origen de un concepto crítico al tradicional, y si bien la actual dinámica de las RRII continúa estando delineada por el poder, y siguen presentes, quizá como nunca antes, riesgos y amenazas a la seguridad arraigadas al conflicto y a la militarización, la seguridad humana ha mantenido el interés en muchos sectores sociales por considerarse una alternativa de seguridad que conlleve precisamente a la seguridad de las personas.

\section{REFERENCIAS}

Alvear, S. (2001). "Seguridad Humana: la persona como objetivo privilegiado de las políticas públicas nacionales e internacionales". En Rojas, F. y Goucha, M. (editores); Seguridad Humana, Prevención de Conflictos y Paz. Chile, UNESCO-FLACSO.

AWID (2008). "Las mujeres y el genocidio en Ruanda: lo que no se dice". Asociación para los derechos de la mujer y el desarrollo. Recuperado de http://www.awid.org/esl/Temas-yAnalisis/Library/Las-mujeres-y-el-genocidio-en-Ruanda-lo-que-no-se-dice

Baena, G. y Balbi, E. (2006) "El potencial del capital emocional. Posibilidades de la seguridad humana". En Seguridad humana y capital emocional. México, Proyecto PAPIME Laboratorios de Estudios del Futuro DGAPA-UNAM, Red Escenarios y Estrategia en América Latina MP AC/UNU, Nodo Futuro México, Metadata Consultoría y Servicios de Comunicación S.C.

CHS (2003); Final Report of the Commission on Human Security. Human Security Now. New York, Commission on Human Security.

Dahlgren, H. (1992) "Un mundo, una responsabilidad común". Recuperado de http://www. nuso.org/upload/articulos/2122_1.pdf

Fernández. J. (2005); Seguridad humana. Barcelona. Recuperado de http://www.corteidh.or.cr/ tablas/r27406.pdf

Galtung, J. (1984) ¿Hay alternativas? Cuatro caminos hacia la paz y la seguridad. Madrid, Tecnos.

Gomáriz, E. (2004). "La doctrina de la seguridad democrática y el impacto paradigmático de la crisis global". En Rosas, C. (compiladora) Seguridad hemisférica e inseguridad global. México, Ministerio de Asuntos Exteriores de Canadá, Universidad Nacional Autónoma de México. 
Knigth, A. (2003) "Las Naciones Unidas y la seguridad internacional". En Rosas, C. (compiladora), Cooperación y conflicto en las Américas. Seguridad hemisférica: un largo y sinuoso camino, México, Universidad Nacional Autónoma de México.

Kornblitch, M. (2001). "Seguridad Humana, redefinición y retos para América Latina y el Caribe". En Rojas, F. y Goucha, M. (editores); Seguridad Humana, Prevención de Conflictos y Paz. Chile, uNESCO-FLACSO.

Márquez, R. (1998). "Esferas de seguridad y linderos del corazón en las tinieblas" en Revista de Administración Pública, núm. 98, México, Instituto Nacional de Administración Pública.

Moller, B. (2000). "Seguridad nacional, societal y humana: el marco general y el caso de los Balcanes", Análisis, año 15, núm. 4, Fasoc.

Nef, J. (2001). "Seguridad humana y vulnerabilidad mutua". En Rojas, F. y Goucha, M. (editores); Seguridad Humana, Prevención de Conflictos y Paz. Chile, UNESCO-FLACSO.

Nef, J. (2003). "Human Security and Mutual Vulnerability", en Goucha, M. y Rojas, F. Human Security, Conflict Prevention and Peace for Latin America and the Caribbean. Santiago, UNESCO-FLACSO.

Olof Palme (1982). Reporte de la Comisión Independiente sobre Asuntos de Desarme y Seguridad. Seguridad mundial. Un programa para el desarme. México, Lasser Press.

OMS-OPS (2002). Informe mundial sobre la violencia y la salud 2002. Washington, Organización Panamericana de la Salud-Organización Mundial de Salud.

Oswald, U. y Gunter, H. (2009) "Introducción. Globalización y desafíos ambientales cambian la conceptualización de seguridad en América Latina". En Oswald, U. (editora) Reconceptualizar la seguridad en el siglo XXI. México, Centro de Ciencias de la Atmósfera, Centro de Investigaciones Interdisciplinarias en Ciencias y Humanidades, Centro Regional de Investigaciones Multidisciplinarias, Senado de la República, LX Legislatura, AFESPRESS.

Peña, R. (1998). "La nueva dimensión de la seguridad internacional". En Cid. I., compiladora, Compilación de lecturas para la discusión de las relaciones internacionales contemporáneas. México, Universidad Nacional Autónoma de México.

PNUD (1994). Informe sobre Desarrollo Humano 1994. Un programa para la Cumbre Mundial de Desarrollo Social. Nueva York, Programa de Naciones Unidas para el Desarrollo, Naciones Unidas, Oxford University Press. 
(2009). Informe sobre Desarrollo Humano 2009. Superando Barreras: movilidad y desarrollo humanos. México, Programa de Naciones Unidas para el Desarrollo, Mundi Prensa.

Sánchez Noriega, A (2011); "Derechos humanos: legitimidad y seguridad internacional". Ponencia dictada en el XXV Congreso Anual: AMEI: 25 años de análisis de la realidad internacional". México.

UNCTAD (1997). Informe sobre el Comercio y el Desarrollo de 1997, UNCTAD. Recuperado de http://www.unctad.org/templates/Webflyer.asp?docID=3303\&intltemID =2280\&lang=3

UNDP (1992). Human Development Report 1992. Global dimensions of human development. New York, Oxford University Press.

UNTFHS (2009); Human security in theory and practice. Application of the Human Security Concept and the United Nations Trust Fund for Human Security. New York United Nations.

Villanueva, M. (2002) "La seguridad humana: ¿una ampliación del concepto de seguridad global?". En Revista Mexicana de Política Exterior, núm. 59, febrero, México, Instituto Matías Romero.

Zavaleta. S. (2014). "Alcances y límites de la seguridad humana como el marco de acción del Estado para proveer seguridad". En La seguridad humana en México. México, Comisión de Derechos Humanos del Distrito Federal-Universidad Autónoma Metropolitana Xochimilco. En proceso de publicación.

\section{ÍNDICE DE CUADROS}

PÁG.

$\begin{array}{ll}\text { Cuadro 1. Desigualdad en los noventas } & 72\end{array}$

Cuadro 2. Estado de la pobreza. Millones de personas.1990-1995 73

Cuadro 3. Disparidades en el PIB (1960/1990) Índice Norte $=100$

Cuadro 4. Componentes e indicadores de la seguridad humana 83 